\title{
Shifts in the Foundation: The Continual Modification and Generalization of Axioms and the Search for the Mathematical Principles that Underlie our Reality
}

\author{
Dr. Indrajit Patra ${ }^{a}$ \\ A \\ An Independent Researcher
}

Article History: Received: 11 January 2021; Accepted: 27 February 2021; Published online: 5 April 2021

\begin{abstract}
The study shall seek to explore the deep, underlying correspondence between the mathematical world of pure numbers and our physical reality. The study begins by pointing out that while the familiar, one-dimensional real numbers quantify many aspects of our day-to-day reality, complex numbers provide the mathematical foundations of quantum mechanics and also describe the behavior of more complicated quantum networks and multi-party correlations, and quaternions underlie Einsteinian special theory of relativity, and then poses the question whether the octonions could play a similar role in constructing a grander theory of our universe. The study then points out that by increasing the level of abstraction and generalization of axiomatic assumptions, we could construct a more powerful number system based on octonions, the seditions, or even other hypercomplex numbers so that we may more accurately describe the universe in its totality.
\end{abstract}

Keywords: mathematical structures, mathematical universe, fine-tuning, number system, real numbers, complex numbers, quaternions, octonions, seditions, string theory, symmetry, multiverse, division algebras, Standard Model of particle physics, supersymmetry

\section{Introduction}

What is it that breathes fire into the equations and makes a universe for them to describe? - Stephen Hawking

As this article attempts to take a closer look at the various ways in which the macroscopic and microscopic world of physical reality and the mathematical world of pure numbers interact, influence and inspire each other, it is of great importance that we try to understand the intrinsically mathematical nature of our own universe.

Many fundamental constants of nature and initial conditions of the universe do indeed appear as being exquisitely fine-tuned to an unimaginably high degree of precision. Parameters like dimensionality of the universe, matter-antimatter asymmetry, spontaneous breaking of the electroweak symmetry and bosons acquiring their masses through the Higgs mechanism, various cosmological features like cosmic mean density, Hubble and cosmological constant, curvature etc., values of various coupling constants, fine-tuning of a multitude of nuclear processes such as the deuterium bottleneck, the triple-alpha process of stellar burning, and nucleon-nucleon binding etc. to name a few - are some of the most striking examples of fine-tuning operating in the cosmic scale. Max Tegmark, in his Our Mathematical Universe, explores the controversial idea that "the ultimate reality is purely mathematical, demoting familiar notions such as randomness, complexity, and even change to the status of illusions, and implying that there's a fourth and ultimate level of parallel universes" (13). According to his classification of multiverses, the Level IV multiverse is the ultimate, ensemble multiverse where mathematics does not describe any external physical reality, but the reality in its grandest form itself is mathematics, and our physical world or the different branches of parallel universes in the Level III multiverse becomes a giant mathematical object in the Level IV multiverse that encompasses all mathematical objects. In Tegmark's words, "the Level IV parallel universes dance to the tunes of different equations, corresponding to different mathematical structures" (Our Mathematical Universe 321). Various other books like van Fraassen's Laws and Symmetry (1989), Victor Stenger's The Comprehensible Cosmos: Where Do the Laws of Physics Come From? (2006), Brian Greene's The Hidden Reality: Parallel Universes and the Deep Laws of the Cosmos (2011), Yanofsky's The Outer Limits of Reason: What Science, Mathematics, and Logic Cannot Tell Us (2013), and Dray and Manogue's The Geometry of the Octonions (2015) - attempt to delve deep in the discussion of the relationship between mathematics and physical reality.

Barrow and Tipler (1986), Leslie (1989), Davies (2006), Ellis (2007), and Barnes (2012) have pointed out this fine-tuning aspect of cosmos. These examples of fine-tuning therefore make us wonder at the intrinsically mathematical nature of our cosmos. One example is the so-called 'Beryllium bottleneck' and the associated phenomenon of Hoyle state resonance. Now, the process of making carbon starts with two $4 \mathrm{He}$ nuclei colliding together to form $8 \mathrm{Be}$, upon which a third $4 \mathrm{He}$ nucleus fuses with $8 \mathrm{Be}$ to produce $12 \mathrm{C}$. It is from this $12 \mathrm{C}, 16 \mathrm{O}$ is made after combining another $4 \mathrm{He}$ nucleus. Now as the $8 \mathrm{Be}$ isotope is extremely unstable, the second step must occur pretty rapidly, and as Hoyle predicted, in the absence of the exquisitely fine-tuned energy level in $12 \mathrm{C}$, this process would be too inefficient in creating sufficient amount of carbon. Also, the mass difference between protons and neutrons, or, between the down quark and the up quark is also extremely fine-tuned. Also, speaking of the initial conditions of our universe, we see that the number Q, or size of primordial inhomogeneities, or 'ripples' is also exquisitely fine-tuned. The value of $Q$ is around 0.00001 , or one part in a 100,000. If this value was $1 / 10$ th of 
his present value, then no galaxies could have emerged and consequently no stars and planets, and if, on the other hand, it had been 10 times larger than its present value, all the matter contents of our universe would have collapsed to form only black holes, and so no galaxies, star or planets would arise. Also, equally important is the number $\Omega$, which is defined as the ratio between the current matter density in the Universe and the critical density. If $\Omega \leq 1$, then the universe would continue to expand forever, whereas if $\Omega>1$ it would undergo a tremendous cosmic collapse. Thus $\Omega=1$ is definitely a critical number and the current value is around $\Omega \approx 1$. The density of our universe is $\Omega$ tot $=\rho / \rho$ crit $=1.0002 \pm 0.0026$ which is tantalizingly close to its critical density $\rho$ crit $=3 \mathrm{H} 02 / 8 \pi \mathrm{GN}$ where the curvature of the cosmos also contributes with its value $\Omega \mathrm{k}=0.000 \pm 0.005$. So, unless the number $\Omega$ had the right value from the dawn of time to a precision of almost 55 decimal places, it would not have been possible for the number $\Omega$ to retain and maintain its critical value for few billions of years. Grinbaum (2012) discusses this problem in more details. Also, the smallness of cosmological constant compared to the enormous value of Planck energy is another example in fine-tuning. As the natural energy scale of gravitation, the Planck scale is somewhat close to $\mathrm{mPl}=\left(\mathrm{h}^{-} \mathrm{c} / \mathrm{GN}\right) 1 / 2=1.2 \times 1019 \mathrm{GeV} / \mathrm{c} 2$, and the cosmological constant or dark energy density $\Lambda$ is close to $(10 \mathrm{meV}) 4 \sim(10-30 \mathrm{mPl}) 4=10-120 \mathrm{mPl} 4$. The cosmological constant is also extremely tiny when compared to the vacuum expectation value of the Higgs field which has a value of about mH4 (100 GeV)4 1052 L.

Even though physicists like Liddle and Lyth 2000 and Weinberg 2008 propose cosmic inflation to solve the fine-tuning problem for $\Omega$, we find that even the parameters in any theory of inflation have to be fine-tuned to few millions of decimal places (Carroll and Tam ,2010). Also, as Penrose points out, the initial conditions of our universe must have been fine-tuned with a precision of one to $10^{\wedge} 10^{\wedge} 123$. There are as many as 26 dimensionless constants which further point towards the extremely fine-tuned, and intrinsically mathematical nature of our universe. There is the famous fine-structure constant, or the strength of the electromagnetic interaction, which id more specifically the ratio of the charge of an elementary particle such as an electron squared to Planck's constant and the speed of light. The number is $\approx 1 / 137.036$. Then, the strong coupling constant is there to determine the strength of the force that glues protons and neutrons together. We require as many as 15 dimensionless constants to describe all the known masses in our universe. The masses of the six quarks, six leptons, the W, Z, and the Higgs bosons also call for the invention of some fundamental theory which might describe the process through which these masses arise and take their extremely precise values. Then we have four quark mixing parameters and three neutrino mixing parameters for the three types of neutrino species all of which possess the same quantum number. Finally, there is the cosmological constant $\Lambda$ which is the vacuum energy density of space, that is a consequence of Einstein's field equations of general relativity. The ideas of dark energy and quintessence are invoked to describe the nature of this cosmological constant.

\section{The Relationship of Fundamental Physical properties with Pure Mathematics:}

The relationship of fundamental physical properties with pure mathematics has intrigued many mathematicians and physicists. Recently, it is suspected that the entire array of fundamental forces and particles actually spring from the properties of eight-dimensional numbers named "octonions." Speaking of number system, the most familiar numbers are definitely real numbers, which includes rational numbers such as integers $(-2,-1,0,1,2)$, fractions $(1 / 2,1 / 3,0.8,2.5)$ and irrational numbers such as $\sqrt{3}, \sqrt{5}, \pi(22 / 7$ or $3.14159265358979 \ldots)$, Euler's Number, e (2.71828182845904...), Golden ratio, $\varphi(1.61803398874989 \ldots . .$.$) , etc. to name a few. Now, these real$ numbers can be combined in a special way to form "complex numbers," which were first found during the 16thcentury Italy, and these numbers can also be imagined to lie like coordinates on a 2-D plane. It is by combining a real Number and an imaginary number that we derive a complex number. In the example, a+bi, a can be thought of as the 'real part,' and 'bi' the imaginary part, since the 'i' possesses a value of square root of negative one. Also, we can think of such acts as translating and rotating positions around the plane as equivalent to the performing of such basic operations like adding, subtracting, multiplying and dividing. Now, the complex numbers, when paired in a particular way, can give rise to the 4-D "quaternions". Any single point in 4-D space with Cartesian coordinates $(\mathrm{t}, \mathrm{x}, \mathrm{y}, \mathrm{z})$ may be represented by a quaternion $\mathrm{P}=\mathrm{t}+\mathrm{xi}+\mathrm{yj}+\mathrm{zk}$. A general 4D rotation can be split into left- and right-isoclinic factors. If complex algebra can be thought of as 2-D, quaternion algebra can be thought of as occurring in 4-D plane: $\mathrm{i}^{\wedge} 2=\mathrm{j}^{\wedge} 2=\mathrm{k}^{\wedge} 2=\mathrm{ijk}=-1$. Here, using $1, \mathrm{i}, \mathrm{j}, \mathrm{k}$ as the base, we see how a 4-axis space is created. Quaternions are pretty easy to interpolate which implies that applying two rotations, it is possible to transform one into the other in a smooth way without running into any problems. One needs four numbers to represent a quaternion, and this, in turn, is related to the number of some special 2D planes that seem to exist in $3 \mathrm{D}$ space. In the 4-normed division algebras over the reals, where division by non-zero elements become possible, we see that besides the real numbers, there are also the complex numbers, quaternions, and a non-associative number system called octonions comprise the system. When we apply the Cayley-Dickson construction, we see that at each step, the number of dimensions gets doubled in a geometric progression: $1,2,4,8$. This is why the dimension number 3 remains absent. Another aspect that will continue to become ever more important as we will move up or down the Cayley-Dickson ladder is that with each progress, we tend to lose certain properties while gaining some. When we move up from the real plane to the complex numbers plane, we lose certain order; whereas when we move up to the plane of quaternions, we lose the property of commutativity; and by going to the level of the octonions, we lose associativity; and by moving up to the level of sedonions we lose all properties related to an alternative or a division algebra. The mathematician Cohl Furey has built upon the works of Murat Günaydin 
to unfurl the underlying relationship between fundamental physics and pure mathematics. Many continue to suspect that the eight-dimensional numbers called the 'octonions' seem to give rise to all the fundamental forces and particles. John Graves showed that we can derive octonions from combining certain pairs of quaternions in a particular way and it is the octonion numbers that define coordinates in an abstract 8-dimensional space. Hamilton, the Irish mathematician from whose name the "Hamiltonian" operator in classical and quantum mechanics is derived, first sought to ascend to the complex plane by adding an imaginary $\mathrm{j}$ axis. But working on three dimensions proved to be a highly critical challenge as he faced in his endeavor numerous unforeseen obstacles. Most important of them all is that in the complex plane, operations such as multiplication produce rotations. It became increasingly hard for Hamilton to define multiplication on a 3-D plane, since there was hardly any opposing division to return any meaningful answer. As Charlie Wood writes in Wired, "To see what makes 3-D rotation so much harder, compare turning a steering wheel with spinning a globe. All the points on the wheel move together in the same way, so they're being multiplied by the same (complex) number. But points on the globe move fastest around the equator and slower as you move north or south. Crucially, the poles don't change at all. If 3-D rotations worked like 2-D rotations, Baez explained, every point would move" ("Meet the Four-Dimensional Numbers That Led to Modern Algebra"). However, it is by adding three imaginary axes, i, j and k, with the real number line 'a', Hamilton found that these new numbers appear to function like arrows in 4-D space, and he named them "quaternions." These 'arrow'-like quaternions could also be rotated on the 3-D plane. Hamilton thought of ways in which he could simplify these quaternions where he could just keep the real part, a, equal to zero and let its imaginary components $\mathrm{i}, \mathrm{j}$ and $\mathrm{k}$ function normally. For these three imaginary components, Hamilton coined the notation "vector." The act of rotating a 3-D vector became equivalent to the multiplication of it by a pair of 4-D quaternions which also happened to contain information about the direction and degree of rotation. One could perform almost all the operations effortlessly with the quaternions what he/she can perform with the real and complex numbers. However, in case of quaternions, ordering matters. Whereas $4 \times 5$ and $5 \times 4$ both yield 20 , when it comes to multiplication with quaternion, order matters, and this behavior used to be encountered frequently in ways in which objects rotate but never found in the world of numbers. numbers before, even though it reflects how everyday objects rotate. "Place your phone face-up on a flat surface, for example. Spin it 90 degrees to the left, and then flip it away from you. Note which way the camera points. Returning to the original position, flip it away from you first and then turn it to the left second. See how the camera points to the right instead? This initially alarming property, known as non-commutativity, turns out to be a feature the quaternions share with reality" (Wood, "Meet the FourDimensional Numbers").

However, during 1898, it becomes quite clear that reals, complex numbers, quaternions and octonions are the only kinds of numbers on which we can apply addition, subtraction, multiplication and division. Thus, the field of "division algebras" became the mathematical bedrock for 20th-century physics, where real numbers appear universally, complex numbers providing the mathematical foundations for the math of quantum mechanics, and quaternions becoming useful tools in Einstein's Special Theory of Relativity. So, the question whether the octonions could hold the foundational secrets of our reality becomes important. Pierre Ramond, a particle physicist and string theorist at the University of Florida states, "Octonions are to physics what the Sirens were to Ulysses". Günaydin and his advisor Feza Gürsey discovered a connection between the octonions and the strong nuclear force in 1973. However, with the advent of the Standard Model of particle physics, the focus of most of the researchers shifted to the results obtained from experiments performed in the collisions of high energy colliders and related experiments while also adding extra particles with the known ones in the hope of discovering an ultimate theory of everything. As Latham Boyle, a theoretical physicist at the Perimeter Institute of Theoretical Physics in Waterloo, Canada, feels that the physicists hoped "that the next bit of progress will come from some new pieces being dropped onto the table, [rather than] from thinking harder about the pieces we already have."

In recent times, we see researchers like Furey attempting to build on the works of Günaydin and Gürsey in the hope of constructing an octonionic model of both the strong and electromagnetic forces. Professor Günaydin had through his researches sought to uncover some deep, underlying connections between String Theory, M-theory and supergravity and octonions themselves which parallels the attempts of other researchers in the field of Quantum Gravity or theories that attempt to unify gravity with the other three fundamental forces of pour universe. Furey has devised several ways in which the octonions could be connected to the Standard Model but has not attempted to unite gravity within the octonionic paradigm. There is a tantalizing indication that the division algebras along with the octonions could very well be a part of the mathematical foundations of our reality. In order to unify the fundamental forces and the particles with the octonionic model, one needs to construct an elegant and simple model, and the ways in which one could combine the octonions and other division algebras could open the door for many mathematical revolutions in the near future. Professor Michael Duff, a string theorist has also researched on the octonions' role in string theory.

In every step of the ladder, as one moves from real numbers to complex numbers, and then to quaternions and to octonions, the numbers of dimensions are doubled and certain properties get also lost. In case of the real numbers, one can arrange them from smallest to largest, while in the complex plane there's no such way for arranging numbers from smallest to largest. Next, in case of quaternions, we lose the property of commutativity, as for these numbers, $\mathrm{a} \times \mathrm{b}$ does not equal $\mathrm{b} \times \mathrm{a}$. Since quaternions exist in a 4-D plane, switching the order of rotations in more than two dimensions can also shift the position of the numbers. In case of the octonions all 
associativity is lost where $(\mathrm{a} \times \mathrm{b}) \times \mathrm{c}$ no longer implies the same thing as $\mathrm{a} \times(\mathrm{b} \times \mathrm{c})$. As John Baez, a renowned mathematical physicist states, "Nonassociative things are strongly disliked by mathematicians. Because while it's very easy to imagine noncommutative situations - putting on shoes then socks is different from socks then shoes - it's very difficult to think of a nonassociative situation." The nonassociative nature of the octonions make it extremely difficult to combine it with the dozens of particles and anti-particles in the Standard Model theory. The Standard Model is built on the notion of three symmetry groups dubbed as SU(3), SU(2) and U(1), which correspond to the strong, weak and electromagnetic forces, respectively. The Standard Model and its three symmetry groups contain subsets of particles which upon being interchanged leave the equations unchanged. The model contains six types of quarks and the six anti-quarks, the eight gluons, the three charged leptons and three antileptons, the three neutrinos and antineutrinos, the photon, the $\mathrm{W}$ and $\mathrm{Z}$ bosons, and the Higgs boson. Gravity is not included in this model and exists separately.

Just as we find the four corners of a square giving rise to a symmetry of 90-degree rotations, similarly in the Standard Model Theory of particles, sets of particles with their unique handedness, arrangement of specific charges and division into three generations - all realize the symmetry group known as $\mathrm{SU}(3) \times \mathrm{SU}(2) \times \mathrm{U}(1)$. There is a chance that this model points towards some yet undiscovered theoretical structure which unifies all the particles and forces in a coherent and comprehensive framework and in this regard, the octonions could come handy in the construction of such a complete theoretical framework. If one imagines the particles to be rotating in a 4-D spacetime, quaternions could represent the way these particles translate and rotate in the 4-D. As Furey states, "I realized that the eight degrees of freedom of the octonions could correspond to one generation of particles: one neutrino, one electron, three up quarks and three down quarks." Furey sought to reconstruct the internal properties like charge of the particles using the four division algebras or the Dixon Algebra which is composed of real numbers, complex numbers, quaternions and octonions. The four number systems can form a 64-dimensional space and the components of the subspace when multiplied by other components remain fixed in the subspace which in turn allows the particles to stay particles even when they are rerating, interacting, moving and transforming. This is the ' $\mathrm{R} \otimes \mathrm{C} \otimes \mathrm{H} \otimes \mathrm{O}$ combination' of four number system where $\mathrm{R}$ stands for reals, $\mathrm{C}$ is for complex numbers, $\mathrm{H}$ represents quaternions and $\mathrm{O}$ the octonions. The mathematical ideals embedded in the symmetries of $\mathrm{R} \otimes \mathrm{C} \otimes \mathrm{H} \otimes \mathrm{O}$ system become manifest as particles of the Standard Model.

Under such a transformation, the algebra separates into two parts in which one part is composed of complex numbers combining with quaternions and in the other with octonions: $\mathrm{C} \otimes \mathrm{H}$ and $\mathrm{C} \otimes \mathrm{O}$. Furey has shown in her works how the symmetries of Lorentz group which deals with how particles move and rotate in space-time arise from $\mathrm{C} \otimes \mathrm{H}$ or quaternionic part. Now, the octonionic part $\mathrm{C} \otimes \mathrm{O}$ gives rise to the symmetry group $\mathrm{SU}(3) \times \mathrm{SU}(2)$ $\times \mathrm{U}(1)$, which determine the internal properties of the particles and how the strong, weak and electromagnetic forces, interact with each other.

Also as Günaydin and Gürsey have shown in their early work, the SU(3) can be found inside the octonions. "Consider the base set of octonions, 1, e1, e2, e3, e4, e5, e6 and e7, which are unit distances in eight different orthogonal directions: They respect a group of symmetries called G2, which happens to be one of the rare "exceptional groups" that can't be mathematically classified into other existing symmetry-group families. The octonions' intimate connection to all the exceptional groups and other special mathematical objects has compounded the belief in their importance, convincing the eminent Fields medalist and Abel Prize-winning mathematician Michael Atiyah, for example, that the final theory of nature must be octonionic. "The real theory which we would like to get to," he said in 2010, "should include gravity with all these theories in such a way that gravity is seen to be a consequence of the octonions and the exceptional groups." He added, "It will be hard because we know the octonions are hard, but when you've found it, it should be a beautiful theory, and it should be unique." ("The Octonion Math That Could Underpin Physics"). Günaydin and Gürsey constructed an octonionic model of the strong force operating on a single generation of quarks.

Furey in her paper in The European Physical Journal C, has attempted to include the entire Standard Model symmetry group, $\mathrm{SU}(3) \times \mathrm{SU}(2) \times \mathrm{U}(1)$, generating all the internal properties of such particles as an electron, neutrino and up quarks and their anti-particles. The reason behind the discrete values of electric charge is the value of the whole numbers themselves. Now, instead of capturing a single generation of particles through her algebraic formulation, further work needs to be done to capture the properties of all the three generations of particles. In another paper published in Physical Letters B, Furey uses C $\otimes \mathrm{O}$ to tackle the Standard Model's two other symmetries, namely the SU(3) and U(1). These two are unbroken symmetries while the other symmetry, namely, $\mathrm{SU}(2) \times \mathrm{U}(1)$ can be deconstructed into $\mathrm{U}(1)$ by the Higgs mechanism, through which the particles are endowed with their respective masses. Symmetries can act on all three generations of particles and give rise to even the hypothetical sterile neutrinos. Below is an image describing the number systems in details (courtesy of: Lucy Reading-Ikkanda/Quanta Magazine). 


\section{Four Special Number Systems}

When you add, subtract, multiply or divide the "real numbers" used in everyday life, you always get another real number. Three generalizations of the real numbers also behave in this way. Many physicists believe that all four of these "division algebras" underlie the laws of physics.

\section{R Real numbers}

All the numbers on (1-D) number line.

One defining characteristic of reals

is that their square is never negative.

e.g. $2^{2}=4$ and also $(-2)^{2}=4$

Examples of real numbers:

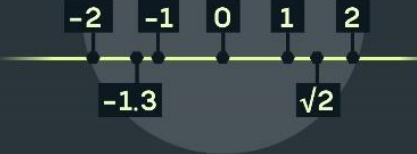

(C) Complex numbers

Reals used in conjunction with an

unconventional "imaginary" unit called i.

One defining characteristic of $i$

is that its square is negative.

ie. $i^{2}=\mathbf{- 1}$

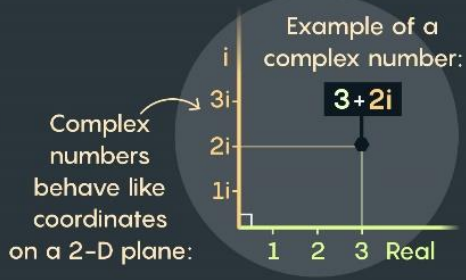

\section{(10) Quaternions}

Reals used in conjunction with three

unconventional units called $\mathrm{i}$, j and $\mathrm{k}$.

Multiplication of quaternions

is noncommutative: Swapping

the order of elements changes

the answer.

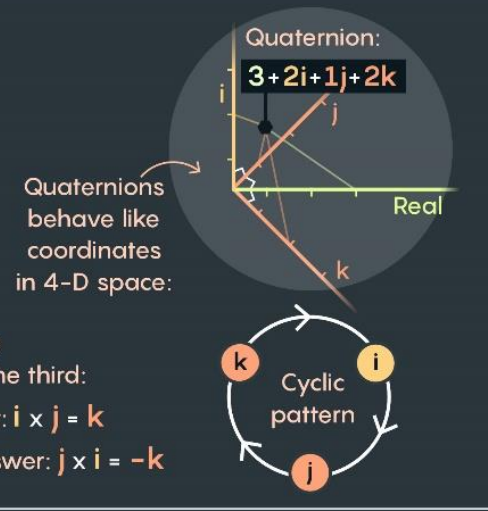

Multiplication follows a cyclic pattern, where

multiplying neighboring elements results in the third:

Moving with arrows gives a positive answer: $\mathbf{i} x \mathbf{j}=\mathbf{k}$

Moving against arrows gives a negative answer: $\mathbf{j} x \mathrm{i}=-\mathrm{k}$

\section{(1) Octonions}

Reals used in conjunction with seven

unconventional units: $e_{1}, e_{2}, e_{3}, e_{4}, e_{5}, e_{6}$

and $e_{7}\left(e_{1}, e_{2}\right.$ and $e_{4}$ are comparable

to the quaternions' i, j and k).

Multiplication of octonions is

nonassociative - it matters

how they are grouped.

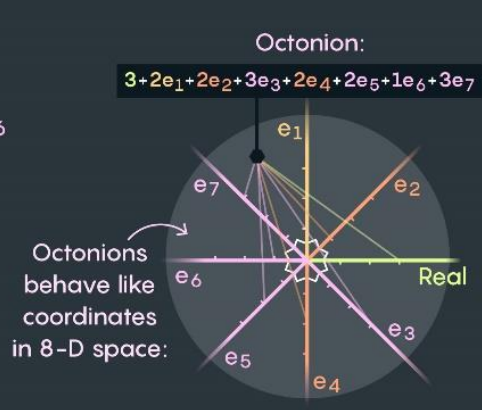

Their multiplication rules are encoded in the

"Fano plane." Multiplying two neighboring elements on a line results in the third element on that same line. Imagine additional lines that close the loop for each group of three elements (e.g., the dashed line).

Moving with arrows gives a positive answer:

e.g. $e_{5} \times e_{2}=e_{3}$ and $e_{6} \times e_{3}=e_{4}$

Moving against arrows gives a negative answer:

e.g. $e_{1} \times e_{7}=-e_{3}$ and $e_{6} \times e_{5}=-e_{1}$

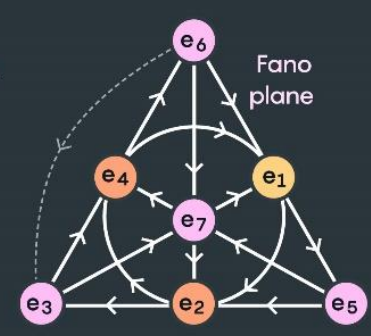

To see their nonassociative property, multiply three elements $e_{5}, e_{2}, e_{4}$

Grouping them like this ... $\left.\left[e_{5} \times e_{2}\right] \times e_{4}=\left(e_{3}\right) \times e_{4}=e_{6}\right\}$ Different

But grouping them like this ... $\left.e_{5} \times\left(e_{2} \times e_{4}\right)=e_{5} \times\left(e_{1}\right)=-e_{6}\right\}$ answers 


\section{Increasing Abstractions and Lessening Axioms:}

Physicists like Rees (1999) has pointed out that the problem of fine-tuning gives us an additional motivation for believing in a theory of Multiverse and the existence of a Multiverse may be a consequence of some form of extrapolation and combination of string theory (Susskind 2005; Schellekens 2013) and theory of cosmic inflation (Liddle and Lyth 2000; Weinberg 2008). There is also the idea of 'emergent multiverse' which Wallace (2012) feels should naturally arise under the framework Many-Worlds or Everettian Interpretation of quantum mechanics. In the quantum mechanical multiverse, all the branches will have the same exact values of the constants and will also obey the same laws of nature.

In a multiverse or even a universe which for the most part lacks structure and order, one could discern and detect structure only if he chooses to look at regions which actually contain the structures. So, physics necessarily deals with predictable phenomena as its primary aim is to make testable and falsifiable predictions. Physics specifically deals with phenomena that involve symmetries and that can yield specific predictions.

The phenomena that are selected by scientists for study must have many different types of symmetry. When a physicist sees a lot of phenomena, she must first determine if these phenomena have symmetry. She performs experiments in different places and at different times. If she achieves the same results, she then studies them to find the underlying cause. In contrast, if her experiments failed to be symmetric, she would ignore them.

Kurt Sundermeyer in his 2014 book Symmetries in Fundamental Physics discusses the role of symmetries in physics ranging "from classical physics to now well-established theories of fundamental interactions, to the latest research on unified theories and quantum gravity". Friedman and Scarr in their 2019 paper titled "Symmetry and Special Relativity" have dealt with "the role of symmetry in the theory of Special Relativity". David Garofalo in his paper titled "Symmetry and the Arrow of Time in Theoretical Black Hole Astrophysics" (2015) considers the case of astrophysical black holes with their manifest properties such as "powerful outflows in active galactic nuclei which deal with macroscopic constituents such as accretion disks, magnetic fields, and black holes" to "identify a parallel between the astrophysical tug-of-war between accretion disks and jets in this model and the time symmetry-breaking of a simple overdamped harmonic oscillator." Laura, et al in their paper "Extended Symmetries at the Black Hole Horizon," have sought to prove that "non-extremal black holes in four-dimensional general relativity exhibit an infinite-dimensional symmetry in their near horizon region." Carlip has attempted to study the underlying two-dimensional conformal symmetry near the horizon of a black hole in his paper titled "Symmetries, Horizons, and Black Hole Entropy."

Although legends like Galileo and Newton first realized and appreciated the role of symmetry in physical phenomena, it took the genius of Albert Einstein to first discover that the laws of physics should remain the same even when the experimenter is moving close to the speed of light. With this symmetry in mind, he was able to compose the laws of special relativity. Einstein's work superseded classical mechanics and proposed the ways in which the laws about how space and time are interrelated and how mass and energy influence and in turn are influenced by the configuration of space and time. Einstein's theory rests on two important axioms, among which the first is that the laws of physics are independent of one's inertial reference frame, and second is that the speed of light in a vacuum always stays a constant. A group known as the Lorentz group captures these principles of symmetries, and from these principles of symmetries Einstein derived the famous $E=m c 2$ along with the rest of the Theory of Special Relativity. Einstein's General Relativity expands and extends the scope of his previous theory to include the phenomenon of acceleration of reference frames from special relativity. However, after its formulation, it soon became clear that in his new theory, the highly cherished principle of conservation of energy seemed to break down. Then it is the mathematician Emmy Noether who through her formulation of the correct symmetry showed that conservation of energy is never violated. G. S. Hall in his book Symmetries and Curvature Structure in General Relativity (2004) presents the theory of classical general relativity from a geometrical viewpoint and deals extensively on the symmetries in general relativity such as "isometries, homotheties, conformal symmetries and affine, projective and curvature collineations." Following Einstein who explored the defining role that symmetries play in fundamental physics, Emmy Noether though her 'Noether's theorem' showed that for every conserved quantity in physics there is a certain kind of corresponding symmetry and so the symmetry and conservation laws are bound together by a deep, underlying connection. This also indirectly influences the values of the fundamental constants of nature, which are the cornerstones of all of the modern physics. So, with every is symmetry there is an associated conservation law and constant. So, the physicist focuses only on those aspects of natural phenomena where symmetries play a crucial role and this is also related to predictability.

Now, if we believe in the multiverse theory, we shall find that it is only in the subset of the universes that possess manifest symmetries where physics works the way we suppose it should work. There is a beautiful analogy between the various number systems and the properties of the universe.

We again need to delve deep into the number system to understand the analogy between number systems and universes. In case of the real numbers, we can easily add, subtract, multiply, and divide these numbers in any way we choose. They are fundamental to virtually every imaginable aspect of science. The real numbers are also always completely ordered which means that given any two random real numbers we can tell which one is bigger and which one is smaller. In the real number line, between any two distinct points on the line, one number will be placed to the right of the other number. Now, next comes the imaginary numbers where the number $i$ has such a property that its square root can be -1 and this is in contrast to any real number whose square root can never be 
negative. An imaginary number thus can be defined as the product of a real number and its imaginary counterpart 'i'. Now, a complex number system results from a combination of a real number and an imaginary number. Noson Yanofsky in the article "Chaos Makes the Multiverse Unnecessary," describes the hierarchy of the number system and its relationship with the multiverse/universe. For the real numbers $r 1$ and $r 2, r 1+r 2 i$ becomes a complex number and since a complex number is constructed from the combination of two real numbers, they can be represented on a 2-dimensional plane. In the complex plane the real number line will reside. We see that this results in the fact that every real number, $\mathrm{r} 1$, can be written as a complex number $\mathrm{r} 1+0 \mathrm{i}$ which is just the number itself with a ' 0 ' complex part of the number. Now, even though one can easily add, subtract, multiply, and divide the complex numbers, in stark contrast to the real numbers, they are never fully ordered. If we take two complex numbers such as, $7+7.5 \mathrm{i}$ and $8-8.5 \mathrm{i}$, we can never tell which one is bigger of the two. Now, we can do some sort of ordering with the complex numbers but this will not involve multiplication, and we will lose certain property. Also, as we have seen in the previous section, similar to the way we can construct the complex numbers from two real numbers, we can also construct the quaternions from two complex numbers. If we take $\mathrm{c} 1=\mathrm{r} 1+\mathrm{r} 2 \mathrm{i}$ and $\mathrm{c} 2=\mathrm{r} 3+\mathrm{r} 4 \mathrm{i}$ as two complex numbers, a quaternion can arise out of these complex numbers in the form of $q=c 1+c 2 j$ where $j$ is a special number. In fact, a quaternion can be written down as $r 1+r 2 i+r 3 j+r 4 k$ where $i, j$, and $k$ are special numbers. Quaternions thus can be thought of as comprising of four real numbers just as a complex number is composed of two real numbers, and also complex number $r 1+r 2 i$ can be written as a quaternion: $r 1+r 2 i+0 j+$ 0k. The quaternions also can be interpreted as a 4-dimensional superset which contains the complex numbers as its two-dimensional subset. Yanofsky also beautifully describes the nature of the quaternions. Quite similar to real and complex numbers, quaternions can be added, subtracted, multiplied, and divided without any hassle. However, in contrast to the complex numbers, quaternions fail to be totally ordered but they possess even less structure than their complex counterparts. The commutative nature of the complex numbers make multiplication of two complex numbers, say c1 and c2 always equivalent irrespective of the order in which they are written. So, c1c2 always equals $\mathrm{c} 2 \mathrm{c} 1$, and this is not the case with the quaternions and so, for two quaternions $\mathrm{q} 1$ and $\mathrm{q} 2 \mathrm{q} 1 \mathrm{q} 2$ is totally different from q2q1.

So, we can interpret octonions as the fundamental system from which other numbers which more structural properties seem to emerge. This is the crux of the process known as "Cayley-Dickson construction," named after the mathematicians Arthur Cayley and Leonard Eugene Dickson which proposes that a new number system can be derived from the original one by doubling the number of the dimension of the original system. The new system with higher dimensions than its predecessor also possesses less structure or demands less axioms than the preceding one. Now, by applying the Cayley-Dickson construction to the quaternions, we the eight-dimensional number system named the octonions. The octonions, too, can be written down as a combination of eight real numbers as $r 1+r 2 i+r 3 j+r 4 k+r 51+r 6 m+r 7 n+r 8 p$. So, if we keep the last four coefficients zero, we can write down a quaternion as a special type of octonion also. The octonions, similarly to the quaternions, are neither fully ordered nor commutative. Further, the octonions are also nonassociative. All the previous number systems contain the property of associativity by virtue of which when the elements a, b, and c, are multiplied, their results a(bc) and (ab)c are always equal. However, the octonions fail to be associative. If we multiply such octonions as o1, o2 and $\mathrm{o} 3$, then their nonassociative nature demands that o1(o2o3) does not equal (o1o2)o3. Also, by keeping the last eight coefficients of the octonion zero, we can derive a sedonion, and for sedenions, it is not possible to divide them even though we can add, subtract or multiply them with ease. One can also construct 32 or even 64dimensional number system from sedenions. Yanofsky attempts to summarize the properties of the five different number systems in the following Venn diagram:

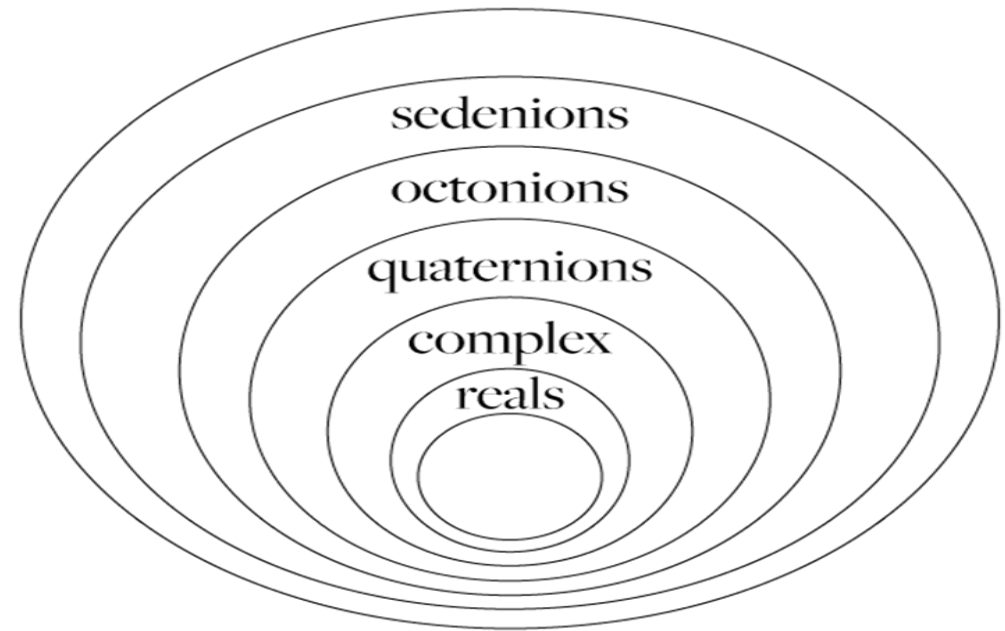

Now, the real numbers are used ubiquitously in physics to denote quantities, measurements, and lengths of physical objects or processes, while the complex numbers assume a central significance in the study of quantum mechanics, and now, in the attempt to unify all the articles and forces in the Standard Model we are beginning to 
see quaternions are being used in special situations. The octonions and the sedenions, too might be used in different branches of physics soon. Leopold Kronecker once stated, "God made the octonions, all else is the work of man." In recent times, there has been a paradigm-shift in our thinking process where we have begun to see complex, quaternions, and octonions as more fundamental than real numbers and octonions appearing increasingly more important in understanding the origin of the hierarchy of number system.

As Yanofsky puts it: Rather than looking at the real numbers as central and the octonions as strange larger number systems, think of the octonions as fundamental and all the other number systems as just special subsets of octonions. The only number system that really exists is the octonions. The octonions contain every number that we will ever need. (And, as we stated earlier, we can do the same trick with the sedenions and even the 64dimensional number system. We shall fix our ideas with the octonions.)

Yanofsky further states that one can find the lost property in the higher dimensional number system in the special type of subfields or subsets of the number system concerned. So, in the subset of the octonions, we can find the property of associative multiplication which does not exist in the multiplication of the octonions themselves. Now, if one selects the octonions in the form of $r 1+r 2 i+r 3 j+r 4 k+01+0 m+0 n+0 p$, the multiplication will be associative similar to what happens with the quaternions. Also, by writing the octonions in the form of $r 1+r 2 i+0 j+0 k+01+0 m+0 n+0 p$, we shall find that the multiplication will be commutative just like it happens with the multiplication of complex numbers. Finally, if one writes the octonions in the form of $r 1$ $+0 \mathrm{i}+0 \mathrm{j}+0 \mathrm{k}+0 \mathrm{l}+0 \mathrm{~m}+0 \mathrm{n}+0 \mathrm{p}$, we will derive a completely ordered number system. So, virtually all the axioms can be thought as residing in the nested forms inside the larger superset of the octonions.

Yanofsky explains it thus: Whenever we have a structure, we can focus on a subset of special elements that satisfies certain properties. Take, for example, any group. We can go through the elements of the group and pick out those $\mathrm{X}$ such that, for all elements $\mathrm{Y}$, we have that $\mathrm{XY}=\mathrm{YX}$. This subset is a commutative (abelian) group. That is, it is a fact that in any group there is a subset that is a commutative group. We simply select those parts that satisfy the axiom and ignore ("bracket out") those that do not. The point we are making is that if a system has a certain structure, special subsets of that system will satisfy more axioms than the starting system. This is similar to what we are doing in physics. We do not look at all phenomena. Rather, we pick out those phenomena that satisfy the requirements of symmetry and predictability. In mathematics, we describe the subset with the axiom that describes it. In physics, we describe the selected subset of phenomena with a law of nature

Yanofsky explains the analogy using the following diagram:

Physics

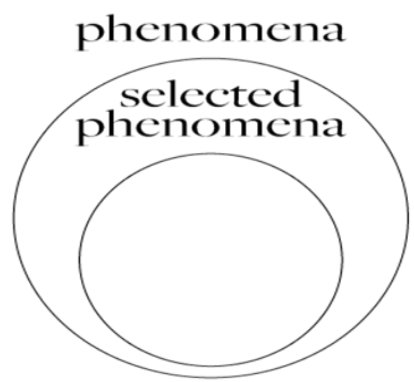

Mathematics

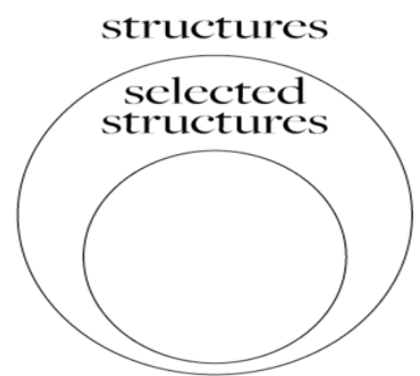

Now, in both mathematics and physics, when we tend to focus our attention to the description of subsets of phenomena rather than the entire phenomena, we discover structures, symmetries and pattern while if we shift our focus towards the vaster scales, we find mathematics becoming more complex than before and physical theories failing to grasp the crux of the problem. However, through their mutual interaction and interrelationship, both mathematics and physics have continued to inspire and influence each other over the ages.

It was with the development in quantum mechanics that the need of complex numbers began to be felt. For describing the distributive nature of quantum mechanics complex numbers appear to play a very crucial role. Also, when Einstein formulated General Relativity, he felt the need to do away with the Euclidean axiom of flatness as being too restrictive and instead chose the non-Euclidean spacetime. In quantum mechanics, the order in which measurement is being done becomes extremely important and so to capture the bizarre beauty of quantum world, we need to discard the cherished principle of commutativity, and here the quaternions become important since they are noncommutative and so, the order of multiplication matters. As quaternions describe rotations in three dimensions, order of multiplication can make a difference to the outcome.

Speaking of the relationship between quantum mechanics and complex numbers we can state that through a special combination of real numbers and a rule to keep them in line can represent the properties of complex numbers and so, quantum mechanics too can be represented via real numbers. Schrödinger himself endeavored to represent his 'real' wavefunction as made of "true wave equation" sans any complex part, i.e., 'i'. In 2008 and 2009, two teams of physicists which include Vértesi and McKague, have attempted to predict the outcome of Bell test by using only real numbers and they have succeeded in doing so. However, various researches are still attempting can prove that quantum mechanical experiments based purely on real numbers may not be enough and that there are complex quantum systems which demand the usage of complex numbers. As Marc-Olivier Renou 
and Nicolas Gisin in their works are attempting to prove that the real number-based version of quantum mechanics cannot go very far in representing the most complex quantum mechanical features. In Bell test experiments, we see that pairs of entangled particles even when are sent far apart, can seem to share information between them, and when physicists measure the results of their measurements on the two particles, they find that the result are correlated in some inexplicable ways. Now, if instead of a single pair of particles we include three pairs of entangled articles where the first pair goes to Alice and Bob, and third pair to Bob and a third party, Charlie, we see that real number-based quantum mechanical description can never fully describe the pattern of correlations in a satisfactory manner. It is only through the use of complex numbers that the correlations in this three-party entanglement can be found. In fact, any attempt to describe such multi-party correlations demands that we do not take recourse to some restrictive class of logic, but instead adopt some less restrictive axiom, and here complex numbers and quaternions become handy since they are more effective in describing the distributive nature of quantum systems.

Also, when Boltzmann and Gibbs began formulating their theories of statistical mechanics, they felt the need to do away with the deterministic mode of thinking. They discovered that the outcomes of their experiments were now no longer bound to binarisitic probabilities where either the event happens $(p(X)=1)$ or it does not $(p(X)=$ $0)$. Rather, in experiments based on statistical mechanics, an outcome $(p(X))$ is no longer seem to be a component of the restrictive finite subset $\{0,1\}$ ), but instead seem to belong to the infinite set $[0,1]$. Max Tegmark in his book Our Mathematical Universe gives us a diagram of the relationship between different number systems and our physical theories.

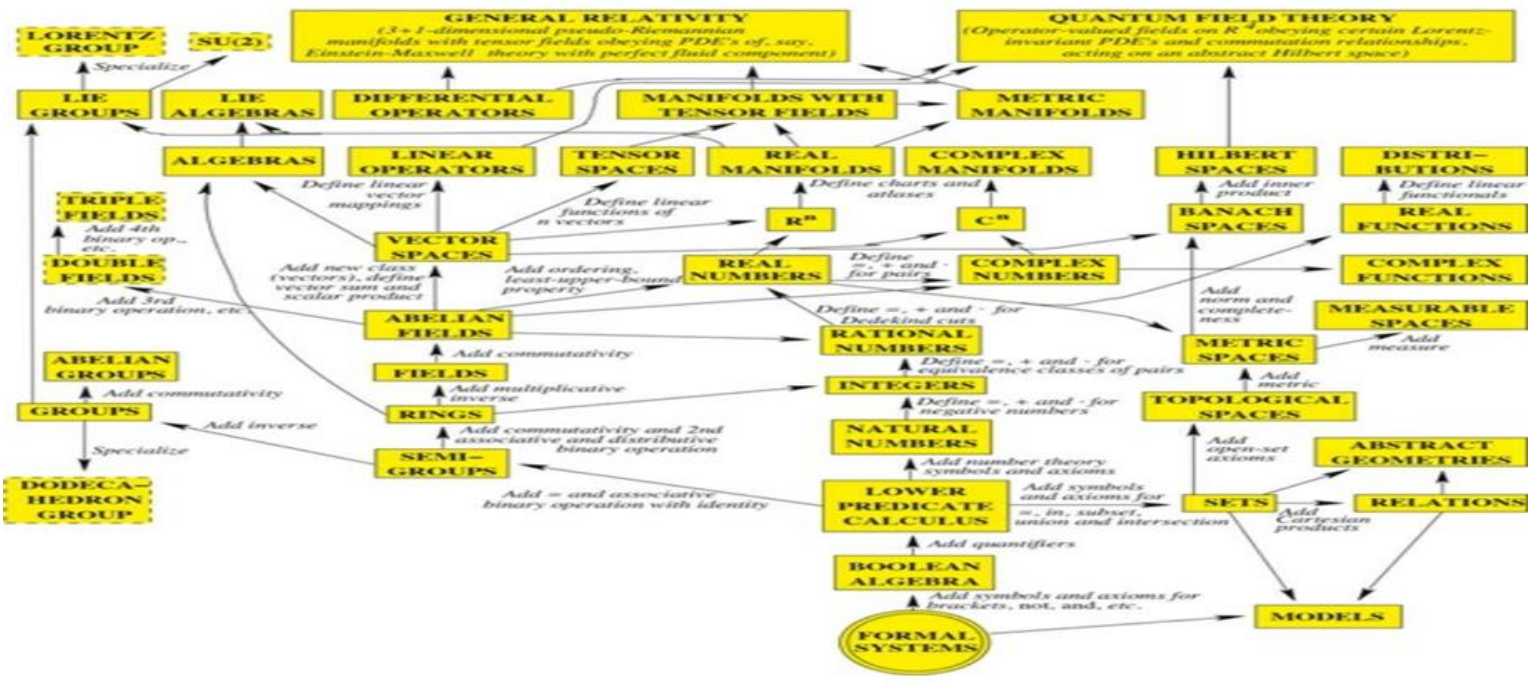

\section{Strings and Dimensionality of our Cosmos:}

String Theory demands that supersymmetry be incorporated in it and this idea of supersymmetry was first formulated during the 1980s. Supersymmetry states that every matter particle such as an electron or neutrino and every force carrying particle such as a photon or gluon possesses a corresponding, twin matter or force mediating particle. Supersymmetry also predicts that the laws of physics would not alter if all the matter and force particles are exchanged with each other. As John C. Baez and John Huerta write: "Imagine viewing the universe in a strange mirror that, rather than interchanging left and right, traded every force particle for a matter particle, and vice versa. 
If supersymmetry is true, if it really describes our universe, this mirror universe would act the same as ours. Even though physicists have not yet found any concrete experimental evidence in support of supersymmetry, the theory is so seductively beautiful, and has led to so much enchanting mathematics, that many physicists hope and expect that it is real" ("The Strangest Numbers in String Theory" 7).

Now, in the standard, 3-dimensionsal version of quantum mechanical description of matter and force particles, we see that spinors describe the wave-like nature of matter particles while vectors represent wave nature of force particles. So, for understanding the nature of particle interactions we have to multiply these two numbers, namely spinors and vectors together.

Now, in a universe where timelike dimension does not exist, we find that in the dimension $1,2,4$, or 8 , a number in a division algebra would be sufficient in describing both matter and force particles that permits addition, subtraction, multiplication and division. In such a timeless universe, in the dimensions $1,2,4$, or 8 , the vectors and spinors can only be real numbers, complex numbers, quaternions or octonions. Supersymmetry thus gives a complete and unifying description of all the matter and forces. However, the description changes radically when we take time into account as at every passing moment, a string despite being a 1-dimensional entity itself actually traces out a 2-dimensional surface. This doubles the dimensions from which supersymmetry emerges as one dimension is required by the string, and one gets added for time. So, we get dimensions 3, 4, 6, or 10 from which supersymmetry emerges instead of from dimensions 1, 2, 4 or 8.

So, Baez and Huerta (2012) state: Coincidentally, string theorists have for years been saying that only 10dimensional versions of the theory are self-consistent. The rest suffer from glitches called anomalies, where computing the same thing in two different ways gives different answers. In anything other than 10 dimensions, string theory breaks down. But 10-dimensional string theory is, as we have just seen, the version of the theory that uses octonions. So, if string theory is right, the octonions are not a useless curiosity: on the contrary, they provide the deep reason why the universe must have 10 dimensions: in 10 dimensions, matter and force particles are embodied in the same type of numbers - the octonions.

Also, there are entities called membranes which are higher dimensional versions of string $\mathrm{s}$ and as time passes, a 2-D brane can trace out a three-dimensional volume in spacetime.

So, instead of adding two dimensions to the 1,2,4 and 8, we have to add three to our standard number of dimensions. So, with the addition of membranes, we find the dimensions 4, 5, 7 and 11 emerging, and in the Mtheory we confront 11 dimensions which naturally calls for an octonionic framework. So, one thing that is becoming increasingly clearer to us is that we need to give up the choice of axioms as we attempt to describe our reality in its grander form:

Paul M. Dirac comments on this necessity of giving up axioms in the following manner:

The steady progress of physics requires for its theoretical formulation a mathematics which get continually more advanced. This is only natural and to be expected. What however was not expected by the scientific workers of the last century was the particular form that the line of advancement of mathematics would take, namely it was expected that mathematics would get more and more complicated, but would rest on a permanent basis of axioms and definitions, while actually the modern physical developments have required a mathematics that continually shifts its foundation and gets more abstract. Non-Euclidean geometry and noncommutative algebra, which were at one time were considered to be purely fictions of the mind and pastimes of logical thinkers, have now been found to be very necessary for the description of general facts of the physical world. It seems likely that this process of increasing abstraction will continue in the future and the advance in physics is to be associated with continual modification and generalisation of the axioms at the base of mathematics rather than with a logical development of any one mathematical scheme on a fixed foundation.

\section{Conclusion}

The study primarily endeavored to explore the analogies between our physical reality and the mathematical world in order to point out the various ways in which the number system in the mathematical world often corresponds to the workings of our physical reality. The study first pointed out the extremely fine-tuned nature of our universe and then pointed out how complex numbers that behave like coordinates on a 2-D plane, the 4-D quaternions, and the octonions that define coordinates in an abstract 8-D space - all can be useful in describing some of the most complex aspects of particle physics and quantum mechanics. Then the study moved on to discuss how mathematical systems with a fewer number of axiomatic assumptions built into them seem to describe the nature of the cosmos in its grandest form imaginable. The larger mathematical structures like the octonionic, the sedenionic, and even hypercomplex numbers from the quaternions to the sedenion number systems should play an increasingly vital role in describing our universe in its totality. Finally, after discussing the mathematical aspects of the dimensionality of string theory the study came to the conclusion that there is indeed a deep, underlying correspondence between the mathematical world of pure abstractions and our physical world.

Author Bio: Dr. Indrajit Patra completed his M.A. in 2011 from Burdwan University and Ph.D. in 2019 from NIT, Durgapur, West Bengal, India. He has published several research articles in various national and international, peer-reviewed journals. His primary areas of interest are posthumanism, transhumanism, virtual reality, the relationship between religion and science, psychoanalysis, and epic poetry. 


\section{References:}

1. Wood, Charlie. "Meet the Four-Dimensional Numbers That Led to Modern Algebra." Wired, WIRED, 9 Sept. 2018, www.wired.com/story/meet-the-four-dimensional-numbers-that-led-to-modern-algebra/. Accessed 8 Mar. 2021.

2. "The Octonion Math That Could Underpin Physics | Quanta Magazine." Quanta Magazine, 2018, www.quantamagazine.org/the-octonion-math-that-could-underpin-physics-20180720/. Accessed 8 Mar. 2021.

3. Renou, Marc-Olivier, et al. "Quantum Physics Needs Complex Numbers." ArXiv.org, 2021, arxiv.org/abs/2101.10873. Accessed 9 Mar. 2021.

4. Yanofsky, Noson S. "Chaos Makes the Multiverse Unnecessary." Nautilus, 29 Nov. 2018, nautil.us/issue/66/clockwork/chaos-makes-the-multiverse-unnecessaryrp?fbclid=IwAR1R981pgPIcVNpdkNkAH-fOc3wMWk3cqzF-KE9WRrlvAfbahKjz-xtuPU4. Accessed 8 Mar. 2021.

5. Yanofsky, Noson S. “Why Mathematics Works so Well.” ArXiv.org, 2015, arxiv.org/abs/1506.08426. Accessed 8 Mar. 2021.

6. Yanofsky, Noson S., and Mark Zelcer. "The Role of Symmetry in Mathematics.” Foundations of Science, vol. 22, no. 3, 7 Mar. 2016, pp. 495-515, link.springer.com/article/10.1007\%2Fs10699-016-9486-7, 10.1007/s10699-016-9486-7. Accessed 9 Mar. 2021.

7. Friedman, Yaakov, and Tzvi Scarr. "Symmetry and Special Relativity." Symmetry, vol. 11, no. 10, 3 Oct. 2019, p. 1235, www.mdpi.com/2073-8994/11/10/1235/htm, 10.3390/sym11101235. Accessed 8 Mar. 2021.

8. Garofalo, David. "Symmetry and the Arrow of Time in Theoretical Black Hole Astrophysics." Journal of Gravity, vol. 2015, 14 July 2015, pp. 1-5, www.hindawi.com/journals/jgrav/2015/530850/, 10.1155/2015/530850. Accessed 8 Mar. 2021.

9. Donnay, Laura, et al. "Extended Symmetries at the Black Hole Horizon." Journal of High Energy Physics, vol. 2016, no. 9, Sept. 2016, link.springer.com/article/10.1007/JHEP09(2016)100, 10.1007/jhep09(2016)100. Accessed 8 Mar. 2021.

10. Carlip, S. "Symmetries, Horizons, and Black Hole Entropy." General Relativity and Gravitation, vol. 39, no. 10, 13 June 2007, pp. 1519-1523, link.springer.com/article/10.1007/s10714-007-0467-6, 10.1007/s10714-007-0467-6. Accessed 8 Mar. 2021.

11. Dirac, Paul M. "Quantised Singularities In The Electromagnetic Field, | Proceedings Of The Royal Society Of London. Series A, Containing Papers Of A Mathematical And Physical Character". 2021, p. ., https://royalsocietypublishing.org/doi/10.1098/rspa.1931.0130. Accessed 8 Mar 2021.

12. "Symmetries and Curvature Structure in General Relativity | World Scientific Lecture Notes in Physics." Worldscientific.com, 2020, www.worldscientific.com/worldscibooks/10.1142/1729. Accessed 8 Mar. 2021.

13. Sundermeyer, Kurt. Symmetries in Fundamental Physics. Cham, Springer International Publishing, 2014, link.springer.com/book/10.1007\%2F978-3-319-06581-6. Accessed 8 Mar. 2021.

14. Baez, John C., and John Huerta. "The Strangest Numbers in String Theory." Scientific American, vol. 304, no. 5, May 2011, pp. 60-65, www.scientificamerican.com/article/the-strangest-numbers-in-stringtheory/, 10.1038/scientificamerican0511-60. Accessed 9 Mar. 2021.

15. “Imaginary Numbers May Be Essential for Describing Reality.” Quanta Magazine, 2021, www.quantamagazine.org/imaginary-numbers-may-be-essential-for-describing-reality-20210303/. Accessed 9 Mar. 2021.

16. Grinbaum, A. (2012). Which fine-tuning problems are fine? Foundations of Physics, 42, 615-631.

17. Liddle, A. R., \& Lyth, D. A. (2000). Cosmological inflation and large-scale structure. Cambridge: Cambridge University Press.

18. Penrose, R. (1979). Singularities and time-asymmetry. In S. W. Hawking \& W. Israel (Eds.), General relativity: An Einstein centenary survey (pp. 581-638). Cambridge: Cambridge University Press.

19. Penrose, R. (2004). The road to reality: A complete guide to the laws of the universe. London: Jonathan Cape.

20. Rees, M. (1999). Just Six Numbers. London: Weidenfeld \& Nicolson.

21. Schellekens, A. N. (2013). Life at the interface of particle physics and string theory. Reviews of Modern Physics, 85, 1491-1540.

22. Susskind, L. (2005). The Cosmic landscape: String theory and the illusion of intelligent design. New York: Little, Brown.

23. Wallace, D. (2012). The Emergent Multiverse: Quantum theory according to the Everett interpretation. Oxford: Oxford University Press.

24. Barrow, J. D., \& Tipler, F. (1986). The anthropic cosmological principle. Oxford: Clarendon Press.

25. Leslie, J. (1989). Universes. London: Routledge.

26. Davies, P. (2006). The Goldilocks dilemma: Why is the universe just right for life? New York: Mariner. 
27. Ellis, G. G. R. (2007). Issues in the philosophy of cosmology. In J. Butterfield \& J. Earman (Eds.), Philosophy of physics Part B (pp. 1183-1286). Amsterdam: Elsevier.

28. Barnes, L. A. (2012). The fine-tuning of the universe for intelligent life. Publications of the Astronomical Society of Australia, 29, 529-556.

29. Weinberg, S. (2008). Cosmology. Oxford: Oxford University Press.

30. Dray, T. \& Manogue, C.A. The Geometry of the Octonions World Scientific Publishing Company, Singapore (2015).

31. Eddington, A.S. The Philosophy of Physical Science Cambridge University Press, New York, NY (1939).

32. Van Fraassen, B.C. Laws and Symmetry Oxford University Press, New York, NY (1989).

33. Greene, B. The Hidden Reality: Parallel Universes and the Deep Laws of the Cosmos Knopf, New York, NY (2011).

34. Stenger, V.J. The Comprehensible Cosmos: Where Do the Laws of Physics Come From? Prometheus Books, Amherst, NY (2006).

35. Tegmark, M. Our Mathematical Universe: My Quest for the Ultimate Nature of Reality Knopf, New York, $N Y$ (2014).

36. Yanofsky, N.S. The Outer Limits of Reason: What Science, Mathematics, and Logic Cannot Tell Us MIT Press, Cambridge, MA (2013).

37. Furey, C. "Three Generations, Two Unbroken Gauge Symmetries, and One Eight-Dimensional Algebra." Physics Letters B, vol. 785, Oct. 2018, pp. 84-89, www.sciencedirect.com/science/article/pii/S0370269318306452, $\quad$ 10.1016/j.physletb.2018.08.032. Accessed 9 Mar. 2021.

38. Furey, C. “Charge Quantization from a Number Operator.” Physics Letters B, vol. 742, Mar. 2015, pp. 195-199, $\quad$ www.sciencedirect.com/science/article/pii/S0370269315000337?via\%3Dihub, 10.1016/j.physletb.2015.01.023. Accessed 9 Mar. 2021. 